\title{
Laparoscopia en oncología urológica
}

\author{
Hernández Fernández C. \\ Servicio de Urología. Hospital Universitario Gregorio Marañón. Madrid.
}

Actas Urol Esp 2006; 30 (5): 437-438

$\mathrm{L}^{\mathrm{a}}$ a cirugía laparoscópica ha supuesto una absoluta revolución en la urología desde su aplicación, que se atribuye a Cortesi ${ }^{1}$ en 1979, aunque un urólogo español Páramo ${ }^{2}$ ya publica tres años antes una experiencia similar en el diagnóstico de un varón con criptorquidia. Pero es sobre todo con la primera nefrectomía realizada por Clayman en $1991^{3}$, donde empieza su aceptación y expansión, aunque de forma lenta y sujeta a múltiples altibajos.

Curiosamente una de las primeras técnicas laparoscópicas, indicadas en la patología tumoral urológica, fue la linfadenectomía de estadiaje en el cáncer de próstata descrita por Schuessler ${ }^{4}$ donde éste autor demostró la viabilidad de la disección ganglionar pélvica y que convirtiéndose en la técnica con la que se iniciaron la mayor parte de los Servicios de Urología que apostaron por la laparoscopia, incluyendo al nuestro.

La aparición por un lado del PSA y por otro el diagnóstico cada vez más precoz del cáncer de próstata dejaron obsoleta la técnica en pocos años.

Es por tanto, a la cirugía renal a la que se vuelven todos los ojos de los grupos pioneros en estas técnicas y en España es C. Rioja ${ }^{5}$ el que publica la primera nefrectomía realizada por vía transperitoneal y C. Hernández el que comunica la primera por un abordaje retroperitoneal ${ }^{6}$, pero aún así han de pasar años para que se empiece a considerar la laparoscopia como un procedimiento quirúrgico indicado en el tratamiento del cáncer renal, todo ello debido a los teóricos problemas de una resección quirúrgica inadecuada, a la aparición de posibles metástasis en los puertos de entrada y a un mayor tiempo operatorio.

La experiencia sólida de algunos grupos demostraron en pocos años la irrelevancia de estos $^{\text {argumentos }}{ }^{7-9}$.
Hoy podemos afirmar que la indicación más frecuente de la cirugía laparoscópica en Urología, la constituyen el tratamiento de los tumores y sobre todo, desde que la escuela francesa de la mano de Bertrand Guillonneau, demuestra la eficacia de la laparoscopia en el tratamiento del cáncer de próstata ${ }^{10}$.

Recientemente, se han publicado cistoprostatectomías radicales con derivación urinaria realizadas con éxito enteramente por laparoscopia, en modelo animal y humano ${ }^{11,12}$, si bien todavía requieren una mayor depuración de la técnica que la haga comparable en términos de tiempo, son cada vez más los grupos que creen que toda la cirugía se puede realizar por laparoscopia.

Es importante también destacar que dos autores españoles Valdivia a nivel experimental en $1992^{13}$ y Sánchez de Badajoz en $1995^{14}$ en la clínica, publican sendos trabajos sobre la cistoprostatectomía, lo que demuestra que la Urología española, a pesar de sus muchas limitaciones, no ha sido ajena a este cambio en el ámbito de la cirugía.

Para que cualquier nueva técnica, en cirugia oncológica, sea considerada como válida ha de cumplir todos los requisitos de reproductividad y eficacia oncológica $\mathrm{y}$, como se ha comentado anteriormente, tiempos quirúrgicos competitivos con la cirugia convencional.

Hoy, a pesar de las dificultades de entrenamiento, los riesgos de diseminación, etc., es cada vez más obvio que la mayor parte de la cirugía oncológica en urología puede y debe hacerse por laparoscopia, como veremos a continuación los tiempos quirúrgicos son similares, el control oncológico también, claramente menores las complicaciones al igual que menor es la estancia hospitalaria y el tiempo de recuperación a la actividad habitual del paciente. 
Se presentan en esta monografia los datos actualizados que se aportaron en la Mesa Redonda del Grupo de Endoscopia, Litiasis y Laparoscopia desarrollada en Viella en 2005.

Junto con los participantes hemos contado con la colaboración del Dr. Octavio Castillo en el tema "Cistectomía laparoscópica".

He de agradecer a todos ellos su desinteresada participación en la preparación de esta monografỉa.

\section{REFERENCIAS}

1. Cortessi N, Ferrari P, Zambardae A. Diagnosis of bilateral abdominal cryptorchidism by laparoscopy. Endoscopy. 1979;8:33-36.

2. Páramo P. Laparoscopia en testículo oculto. Actas Urol Esp. 1976;44:82-91.

3. Clayman RV, Kavoussi LR, Soper NJ, Dierks SM, Meretyk S, Darcy MD, et al. Laparoscopic nephrectomy: initial case report. J Urol. 1991;146:278-282

4. Schuessler WW, Vancaillie TG, Reich H, Griffith DP. Transperitoneal endosurgical lymphadenectomy in patients with localized prostate cancer. J Urol. 1991;145:988-991.

5. Rioja C, Mínguez P, Blas M, Rioja LA. Nefrectomía laparoscópica: caso report. Actas Urol Esp. 1992;16:544-548.

6. Hernández C, Escribano G, Díez Cordero JM, Verdú F, Palacio A, Jara J. Nefrectomía laparoscópica por vía retroperitoneal. Caso report. Actas Urol Esp. 1994;18:619-622.

7. Mc Dougal EM, Clayman RV, Elashry OM. Laparoscopic radical nephrectomy for renal tumor: The Washington University experience. J Urol. 1996;155:1180-1185.
8. Cadeddu JA, Ono Y, Clayman R, Barrett PH, Janetschek G, Fentie DD, et al: Laparoscopic nephrectomy for renal cell cancer: Evaluation of efficacy and safety: A multicenter experience. Urology. 1998;52:773-777.

9 Kavossi LR, Kerbl K, Capelonto CC, Mc Dougall EM, Clayman RV. Laparoscopic nephrectomy for renal neoplas. Urology. 1993;42:603-609.

10. Guillonneau B., Vallancien G. Laparoscopic radical prostatectomy: the Montsouris technique. J Urol. 2000;163: 1643-1649.

11. Gill IS, Fergany A, Klein EA, Kaouk JH, Sung GT, Meraney $\mathrm{AM}$, et al. Laparoscopic radical cystoprostatectomy with ileal conduit performed completely intracorporeally:the inicial 2 cases. Urology. 2000;56(1):26-30.

12. Gill IS, Kaouk JH, Meraney AM, Desai MM, Ulchaker JC, Klein EA, et al. Laparoscopic radical cistectomy and continent orthotopic ileal neobladder performed completely intracorporeally: tehe inicial experience. J Urol. 2002;168:13-18.

13. Valdivia JG, Vitoria A, Rodríguez J, Valle J, Martínez MJ, Whyte O, et al. Cistoprostatovesiculectomía y ureterosigmoidostomía laparoscópica. Modelo experimental. Actas Urol Esp. 1992;16:592-595.

14. Sánchez de Badajoz E, Gallego JL, Reche A, Gutiérrez JM, Jiménez A. Laparoscopic cystectomy and ileal conduit: case report. J Endourol. 1995;9:59-61.

15. Dunn MD, Mc Dougall EM. Renal Physiology: laparoscopic considerations. Urol Clin North Am. 2000;27(4):609-614.

Dr. C. Hernández Fernández

Servicio de Urología. Hospital Gregorio Marañón

Doctor Esquerdo, 46

28007 Madrid

chdez@pulso.com 